\title{
Dynamic Behaviour of Connectionist Speech Recognition with Strong Latency Constraints
}

\author{
Giampiero Salvi \\ KTH (Royal Institute of Technology), \\ Dept. of Speech, Music and Hearing, \\ Lindstedtsv. 24, 10044 Stockholm, Sweden \\ giampi@kth.se
}

\begin{abstract}
This paper describes the use of connectionist techniques in phonetic speech recognition with strong latency constraints. The constraints are imposed by the task of deriving the lip movements of a synthetic face in real time from the speech signal, by feeding the phonetic string into an articulatory synthesiser. Particular attention has been paid to analysing the interaction between the time evolution model learnt by the multi-layer perceptrons and the transition model imposed by the Viterbi decoder, in different latency conditions. Two experiments were conducted in which the time dependencies in the language model (LM) were controlled by a parameter. The results show a strong interaction between the three factors involved, namely the neural network topology, the length of time dependencies in the LM and the decoder latency.
\end{abstract}

Key words: speech recognition, neural network, low latency, non-linear dynamics

\section{Introduction}

This paper describes the use of a hybrid of artificial neural networks/hidden Markov models (ANNs/HMMs) in a speech recognition system with strong latency constraints. The need for such a system arises from the task of classifying speech into a sequence of phonetic/visemic units that can be fed into a rule system to generate synchronised lip movements in a synthetic talking face or avatar (Beskow, 2004). As the aim is to enhance telephone conversation for hearing-impaired people (Karlsson et al., 2003), the total latency allowed between incoming speech and facial animation is limited by the turn taking mechanism to less than $200 \mathrm{~ms}$. This includes the latency in capturing the sound and generating and animating the facial movements. The constraints 
imposed on the recogniser are thus especially demanding if compared to other applications of speech recognition.

In such conditions, and more in general in real-time applications, conventional decoders based on different flavors of the Viterbi algorithm (Viterbi, 1967), can only be used in an approximate fashion. This is because the need for incremental results requires the best-path solution to be based on partial decisions, with limited look-ahead in time. The difference between the standard Viterbi solution and the approximated solution is often called "truncation error". Truncation error in the Viterbi algorithm has been extensively studied for convolutional codes in the area of speech coding (Kwan and Kallel, 1998; Weathers, 1999). There, given the relatively simple nature of the problem, error bounds could be found analytically and confirmed empirically.

In speech recognition, a few empirical studies dealing with this problem can be found in the area of broadcast news recognition/transcription (e.g. Imai et al., 2000; Ljolje et al., 2000). In Ljolje et al. (2000) a system based on incremental hypothesis correction was shown to asymptotically reach the optimal MAP solution. In Robinson et al. (2002) connectionist techniques are employed in the same task. These studies are concerned with large vocabulary word recognition, and have less stringent latency constraints.

The aim of the current study is to analyse the effect of truncation errors at very low latencies (look-ahead $<100 \mathrm{~ms}$ ) in different set-ups of the language model, while keeping phonetic recognition as the main focus for the application we have in mind. In connectionist speech recognition it is of particular interest to study the interaction between the time evolution model learnt by the time delayed or recurrent neural networks and the transition model imposed by the Markov chain, with varying look-ahead lengths.

The first experiment in this study does this by gradually changing the recognition network from a free loop of phones (short time dependencies) to a loop of words with increasing lengths. In the second experiment the language model (LM) is composed of a scaled mixture of a free loop of phones and a forced alignment topology where the time dependencies are as long as the utterance. The gradual modification of the LM is achieved in this second case by changing the mixing parameter. In addition, a confidence measure particularly suitable for connectionist models (Williams and Renals, 1999) is discussed. The reader also is referred to Salvi (2003) for an evaluation of the Synface recogniser in more realistic conditions.

The paper is organised as follows: a formal definition of the problem is given in Section 2, Section 3 introduces the method, Section 4 the data and experimental settings. Results are described in Section 5 and discussed in Section 6 . Section 7 concludes the paper. 
$\mathbf{s}$

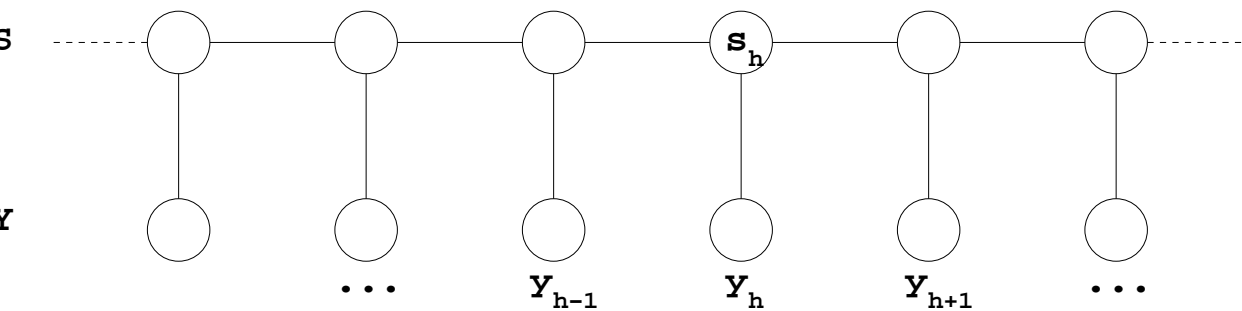

Figure 1. Dependencies in a first order HMM represented as a Bayesian network graph

\section{Problem definition and notation}

\subsection{Speech production in formulae}

The process of speech production could be seen as one of encoding a sequence of symbols $X_{1}^{M}=\left(x_{1}, \cdots, x_{M}\right)$ into a sequence of states $S_{1}^{N}=\left(s_{1}, \cdots, s_{N}\right)$ with an associated output sequence $U_{1}^{T}=\left(u_{1}, \cdots, u_{T}\right)$. In our oversimplified description, $X_{1}^{M}$ could represent phonetic classes, $S_{1}^{N}$ are motivated by the dynamics introduced by articulatory gestures that in turn generate the speech signal $U_{1}^{T}$. Phonetic speech recognition is therefore the process of recovering the original sequence $X_{1}^{M}$ on the base of some features $Y_{1}^{N}=\left(y_{1}, \cdots, y_{N}\right)$ extracted from $U_{1}^{T}$. When the feature extraction procedure is assumed to be given, as in the current study, the distinction between $U$ and $Y$ is not essential. Speech production is then a (stochastic) function of the kind: $P: X \rightarrow Y$. The natural choice for characterising this function is a Markov model $\Theta$ where the states $s_{i}$ are assumed to vary synchronously with the features $y_{j}$, which explains why we indicated the length of $S$ and $Y$ with the same symbol $N$. Besides an a priori term, $\Theta$ is then fully specified by the distribution of state transition probabilities $a_{i j}=P\left(s_{j} \mid s_{i}\right)$ and the likelihood of the data generation given a certain state $b_{i}\left(Y_{h}^{k}\right)=P\left(Y_{h}^{k} \mid s_{i}\right)$. The usual assumption is to consider the latter as local models, in the sense that the state $s_{i}$ at a particular time $h$ influences the observation only at that time $h: P\left(Y_{h}^{k} \mid s_{i}\right)=P\left(y_{h} \mid s_{i}\right)$, as illustrated in Figure 1. In this case, all the information about the dynamic evolution of the process under study is coded in the transition model $a_{i j}$.

\subsection{State-to-output probability estimators}

Robinson (1994) has shown how multi layer perceptrons (MLPs) can be efficient estimators for the a posteriori probabilities $P\left(x_{i} \mid Y_{1}^{n}\right)$ of a certain state $x_{i}$ given an observation $Y_{1}^{n}$. A particularly efficient training scheme uses back propagation through time (Werbos, 1990) with a cross entropy error measure 


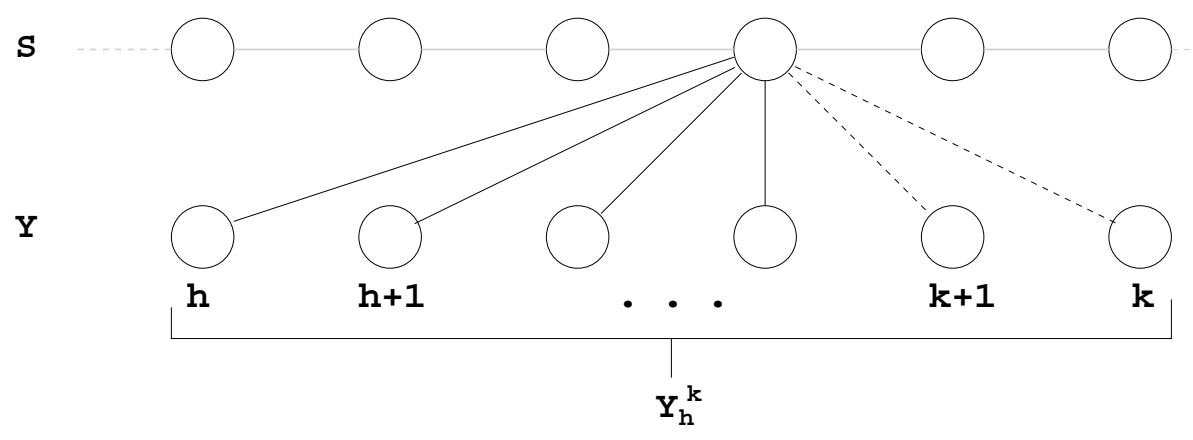

Figure 2. Dependencies introduced by time dependent MLPs.

(Bourlard and Morgan, 1993). If the nonlinearity in the units is in the tanh form, we can write for the state to output probabilities:

$$
P\left(Y_{h}^{k} \mid x_{j}\right)=\frac{P\left(x_{j} \mid Y_{h}^{k}\right) P\left(Y_{h}^{k}\right)}{P\left(x_{j}\right)} \simeq \frac{o_{j}+1}{2} \frac{P\left(Y_{h}^{k}\right)}{P\left(x_{j}\right)}
$$

Where $x_{j}$ is a phonetic class and $o_{j}$ the activity at the output node corresponding to that class. The linear transformation in the formula $\left(\left(o_{j}+1\right) / 2\right)$ is necessary to transform the tanh values, that span from -1 to 1 , into probabilities. In the following we will refer to output activities of the MLP as the linearly transformed outputs that assume values in the range $[0,1] . Y_{h}^{k}$ is the sequence of feature vectors spanning a window of time steps that depends on the dynamic properties of the MLP. In the case of simple feed-forward nets, $Y_{h}^{k}$ reduces to the current frame vector $y_{k}$, while for strict recurrent topologies (RNN), $h=1$ and $k$ is the current frame. This is illustrated in Figure 2 that shows how the dynamic properties of the neural network can introduce dependencies between states and observations that span over a number of time steps. In Ström (1992) a mixture of time delayed and recurrent connections was proposed. In this model the input layer received contributions both from the past and the future frames thanks to time delayed connections with possibly negative delays (represented in the Figure by dashed lines). In this study, only positively delayed connections are considered, in order to reduce the total latency of the system.

\subsection{Interaction between HMM topology and ANN dynamic properties}

Given the probabilistic model $\Theta$, the maximum a posteriori (MAP) solution to the speech recognition problem is the sequence $X_{1}^{M}$ that maximises

$$
P\left(X_{1}^{M} \mid Y_{1}^{N}, \Theta\right)=P\left(x_{1}, \cdots, x_{M} \mid y_{1}, \cdots, y_{N}, \Theta\right)
$$

A more pragmatic solution, provided by the Viterbi algorithm, approximates the sum over all possible state sequences, implicit in Equation 2, with a maxi- 


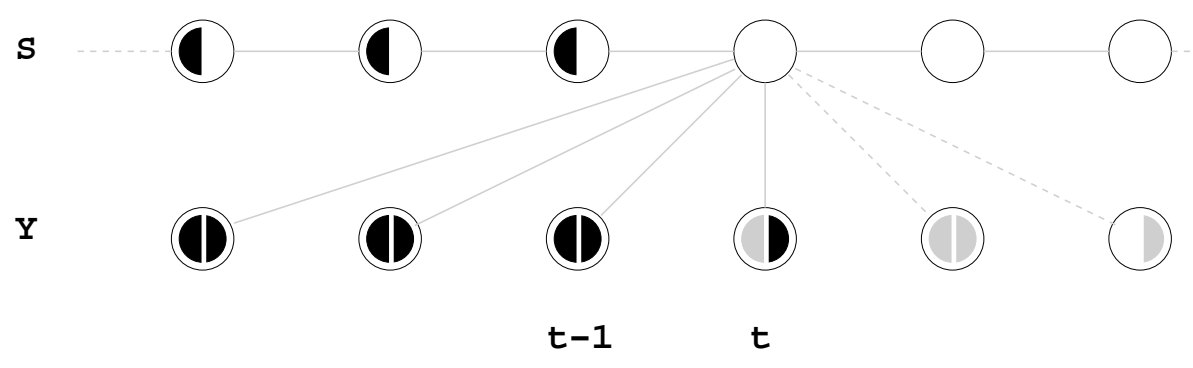

Figure 3. Interaction between the $\delta$ and $b$ terms in Viterbi decoding.

mum operation. Since in our model $X_{1}^{M}$ is fully determined by $S_{1}^{N}$, the recognition problem is equivalent to finding the sequence $S_{1}^{N}$ for which $P\left(S_{1}^{N} \mid Y_{1}^{N}, \Theta\right)$ is maximum. This can be done iteratively according to the Viterbi recursion formula:

$$
\delta_{t}(j)=\max _{i}\left[\delta_{t-1}(i) a_{i j}\right] b_{j}\left(y_{t}\right)
$$

Where $b_{j}\left(y_{t}\right)=P\left(y_{t} \mid x_{j}\right)$ is the likelihood of the current observation given the state and $\delta_{t}(j)$ is the Viterbi accumulator. In practice we substitute to $b_{j}\left(y_{t}\right)$ the estimate of $P\left(Y_{h}^{k} \mid x_{j}\right)$ given by Equation 1 . In the case of recurrent MLPs, $P\left(Y_{h}^{k} \mid x_{j}\right)$ equals $P\left(Y_{1}^{t} \mid x_{j}\right)$ and the information contained by $\delta_{t-1}(i)$ and $b_{j}\left(Y_{1}^{t}\right)$ in the Viterbi recursion formula becomes widely overlapping. Figure 3 illustrates this by indicating which states and observations the two terms in the Viterbi recursion formula depend on. Left semicircles refer to the term $\delta_{t-1}(i)$ and right semicircles to the term $b_{j}\left(Y_{1}^{t}\right)$. Grey semicircles are included if the MLP has negatively delayed connections.

As the two sources of information in the Viterbi recursion are strongly dependent, we expect the evidence brought by their joint contribution to be lower than the sum of each single contribution, as if they were independent.

\subsection{Interaction between HMM topology and look-ahead length}

When truncation is considered, the optimal solution at time step $n$ is the state $s_{n}$ extracted from the sequence $S_{1}^{n+D}=\left(s_{1}, \cdots, s_{n}, \cdots, s_{n+D}\right)$ that maximises $P\left(S_{1}^{n+D} \mid Y_{1}^{n+D}, \Theta\right)$, where $D$ denotes the look-ahead length in time steps. The difference between the two approaches is exemplified in Figure 4. The grid displays the states as a function of time (trellis). The continuous line shows the Viterbi solution, while the dashed and dashed-dotted lines refer to the best path obtained using the partial information up to $t=n+D$ and $t=$ $n+D^{\prime}$, respectively. The figure also illustrates a phenomenon that is common in practice: the influence of an observation at time $t_{1}$ over the result at time $t_{2}$ decays with the distance $D=\left|t_{1}-t_{2}\right|$. In the example the observations in the interval $\left[n+D+1, n+D^{\prime}\right]$ influence the result at time $n$, as prolonging the look-ahead from $D$ to $D^{\prime}$ leads to different results (open and filled squares). 


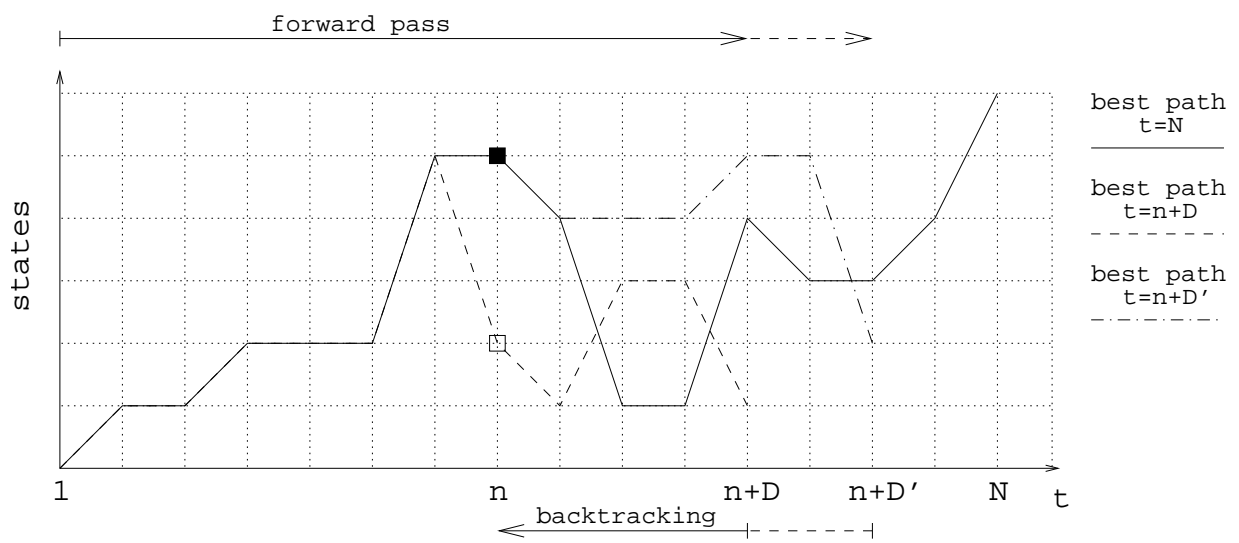

Figure 4. Trellis plot in with three Viterbi paths (varying look-ahead length)

With respect to the solution at time $n$, however, adding the observations in $\left[n+D^{\prime}+1, N\right]$ to the search mechanism does not change the response. As a result the truncated solution will in general asymptotically approach the standard Viterbi solution (filled square in this case) as $D$ increases. The value $D^{*}$ at which the two solutions become indistinguishable depends on the dynamic characteristics of the problem at hand, i.e. on the time correlations in $Y$ and on those imposed by the transition model $\Theta$.

\section{Method}

To evaluate the interaction between the language model, the properties of the probability estimators, and truncation in the Viterbi decoder, three-factor experiments were designed. The factors involved are: the length of time dependencies in the recognition network (language model), the dynamical properties of the probability estimators and the look-ahead length.

\subsection{Language model}

Varying the length of time dependencies in the language model (LM) was simulated in two different ways. In both cases, a different LM is used for each utterance, based on information contained in its transcription.

The first method consists of constructing the recognition network as the union of two topologies with transition weights scaled by a factor $\alpha$ :

$$
\operatorname{LM}(\alpha)=\alpha \mathrm{AL} \cup(1-\alpha) \mathrm{PL}
$$

PL specifies a free loop of the phones included in the transcription for each utterance, while AL is the LM obtained by connecting in a sequence the 


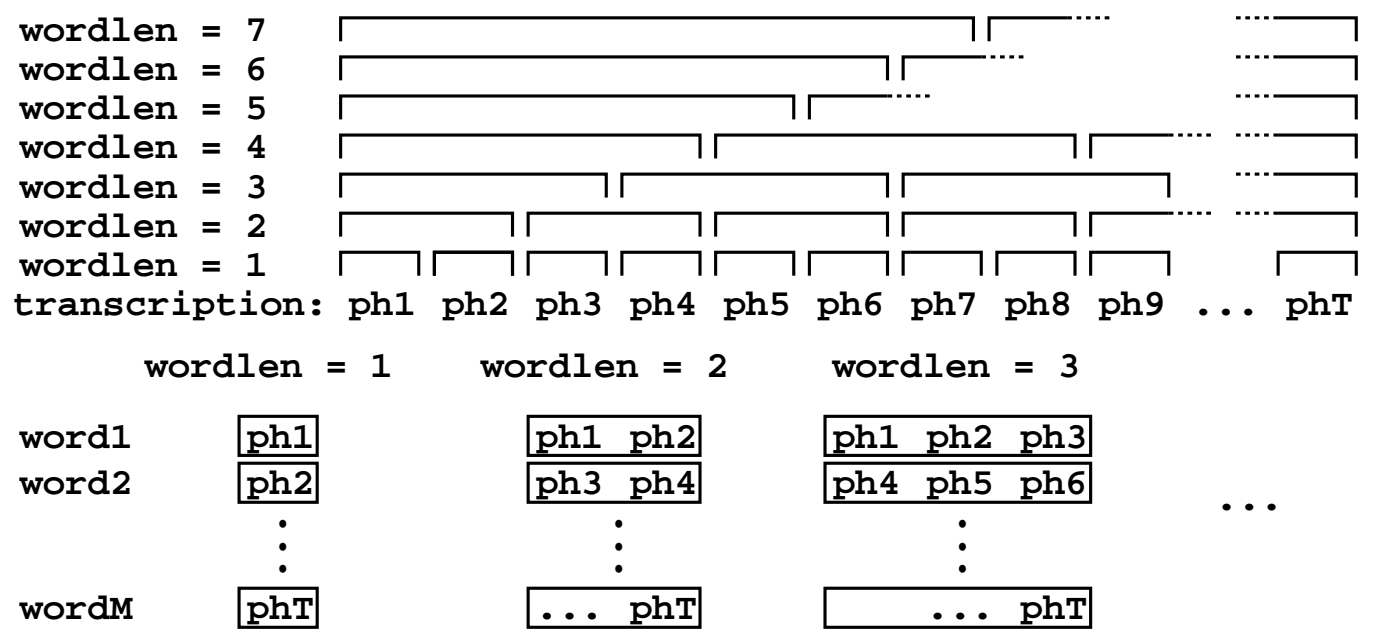

Figure 5. Illustration of the "wordlen test" design: the transcription of each test utterance is spit into words of increasing lengths, that are used in the recognition network.

phones specified by the transcription of each utterance. When $\alpha \rightarrow 0$ the grammar has the short time dependencies of a phone loop, when $\alpha \rightarrow 1$ the time dependencies are as long as the utterance, and the recogniser performs forced alignment. The parameter $\alpha$ assumes seven values in the experiments: $0,0.1,0.3,0.5,0.7,0.9,1$. This design will be referred to as the "alpha test".

In the second method, the LM defines a loop of words, where a word is defined by successively extracting $N$ phones from the transcription of each utterance (see Figure 5). For $N=1$ the LM is again a loop of phones. The parameter $N$ assumes the values from 1 to 7 . To be noted here is that, since each phone is modelled by a three state Markov model, the lower bound to the time dependencies induced by this LM ranges from 3 to 21 frames in the experiments. This design will be referred to as the "wordlen test".

As already noted, the phone-loop condition $(\alpha=0$ or $N=1)$ is obtained in the alpha and wordlen tests by selecting for each test utterance only those phones contained in its transcription. This was necessary to guarantee homogeneity with the other conditions $(\alpha \neq 0$ or $N \neq 1)$, as the main objective of the experiment is to compare the different conditions, rather than computing an absolute performance score. When evaluating the recogniser from the perspective of the application, a loop of all phones should be used. This condition is analysed in Section 5 as a reference.

A factor that turned out to be important in the evaluation of the different LMs, especially in the low-latency condition, is the phone-to-phone transition probability. In the phone-loop condition the transition probability from phone $i$ to phone $j$ is $1 / N$ (the uniform case) where $N$ is the number of phones. In the other conditions, the within-word phone-to-phone transition probability should in principle be 1 , as each phone can only be followed by the next in the 
pronunciation model. This introduces terms that turned out to strongly affect the search mechanism, especially for low-latency conditions. The solution was to keep the same transition probabilities in both cases $(1 / N)$, releasing the constraint of a stochastic grammar (outgoing transition probabilities that sum to 1 ). This is a common practice in speech recognition where multiplicative and additive terms are usually applied to a subset of the transition probabilities, often corresponding to the language model. The aim is however different, as we are not directly interested in tuning the performance of the decoder, but rather in ruling out from the comparison factors that do not depend on the dynamic properties of the LM.

\subsection{Probability estimators}

The second experimental factor is the ability of the state-to-output probability models to express time variations. In this case, similarly to Salvi (2003), three multi layer perceptrons (MLPs) were used with different complexities and dynamic properties. One feed-forward MLP is considered as a static model, while two recurrent MLPs represent models capable of learning the time evolution of the problem (details in Section 4.1).

\subsection{Look-ahead length}

The third experimental factor is the look-ahead length $L$ that can be varied in our decoder. One problem is how to decode the last $L$ frames at the end of each utterance. As every utterance begins and ends with silence, it was suggested in Salvi (2003) to decode the whole test set in a continuous stream, limiting the boundary problem only to the last file. Here, in contrast, each utterance is analysed separately and the result for the last $L$ frames is assumed equal to the best path obtained when the forward phase has reached the end of the utterance. This is a somewhat more standard way of computing the Viterbi solution and was necessary to enable the use of a different language model for each utterance. Five values of the look-ahead length (in frames) were used in the experiments: 1, 3, 5, 10, 20.

\subsection{Scoring method}

The scoring method chosen for this study is frame-by-frame correct classification rate simply computed as the ratio between the number of correctly classified frames and the total number of frames. A correct classification occurs 
when a frame has been assigned the same phonetic class as in the transcription. This method was preferred to the more common minimum edit distance at the symbolic level, because the application we have in mind requires not only correct classification of the speech sounds, but also correct segment alignment. In Section 5, phone-loop results are reported in terms of frame-by-frame correct classification rate, as well as accuracy and percent of correct symbols (Young et al., 2002) in order to compare these scoring methods.

\subsection{Confidence measure}

As noted in Williams and Renals (1999) the property of multi-layer perceptrons to estimate posterior probabilities, as opposed to likelihoods, is advantageous when computing confidence measures of the acoustic models. A simple measure of the acoustic confidence is the per-frame entropy of the $k$ phone class posterior probabilities. Although the entropy measure, in Williams and Renals (1999), is averaged over a number of frames, we will consider a frameby-frame measure. A factor that strongly influences the entropy measure is the choice of the target values during training of the networks. A common practice is to use $0+\epsilon$ and $1-\epsilon$, with small $\epsilon$, as target values, in order to speed up the convergence in the standard back propagation algorithm (Note that when using tanh squashing functions, the limits are internally -1 and 1 , and the above discussion refers to the linearly transformed outputs of the network, see also Equation 1). As a consequence, the networks trained this way are more noisy in the sense that the activities of the inactive output nodes seldom fall below $0+\epsilon$. Strictly speaking, this also gives incorrect posterior probabilities estimates.

To show the effect of the target values on the entropy measure we consider a simplified example. We assume that the activities of the output nodes of the network trained with target values $o_{H}$ (active) and $o_{L}$ (inactive), can only assume those values when the network is excited by new input. In reality the activities take any value in between and sometimes even outside the range $\left[o_{H}, o_{L}\right]$. Even if the network is trained with only one active output node per time step, there will be, during excitation, a number $N_{H}$ of simultaneously active nodes. If we call $N_{L}$ the number of inactive nodes $\left(N_{H}+N_{L}=N\right.$ is the total number of output nodes), then, from the definition of the entropy:

$$
H=-N_{H} b_{H} \log b_{H}-N_{L} b_{L} \log b_{L}
$$

where $b_{H}$ and $b_{L}$ are the normalised activities obtained imposing that the sum of probabilities be 1 :

$$
b_{H}=\frac{o_{H}}{N_{H} O_{H}+N_{L} O_{L}}, \quad b_{L}=\frac{o_{L}}{N_{H} O_{H}+N_{L} O_{L}}
$$




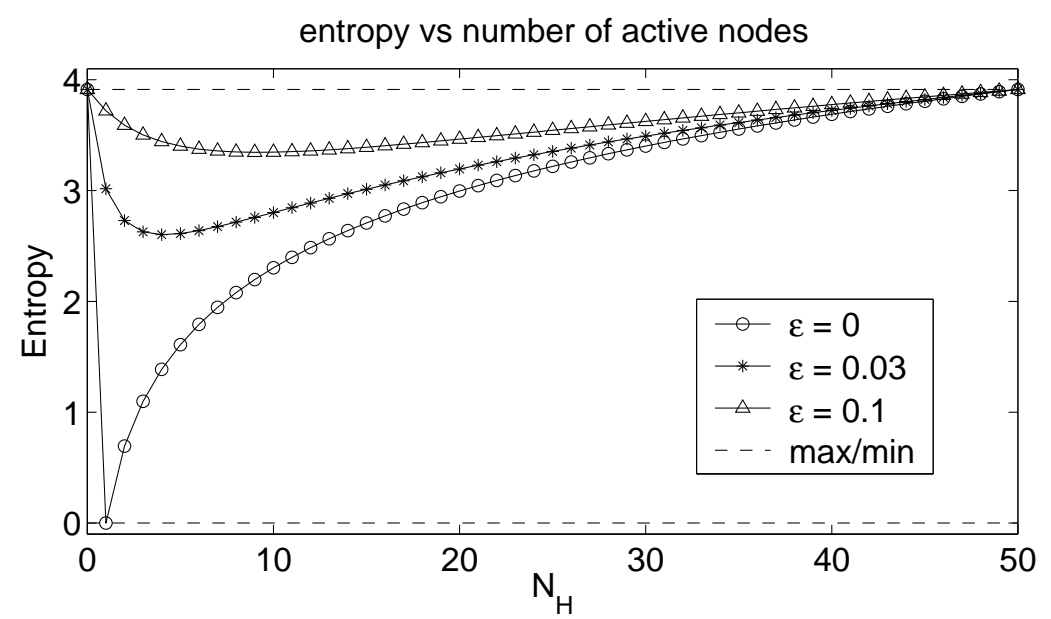

Figure 6. Simulation of entropy of a distribution with $N_{H}$ high levels

In the case of symmetrical values, i.e. $\left(o_{L}, o_{H}\right)=(\epsilon, 1-\epsilon)$, Equation 4 assumes the form:

$$
H=\log \left(N_{H}(1-\epsilon)+N_{L} \epsilon\right)-\frac{N_{H}(1-\epsilon) \log (1-\epsilon)+N_{L} \epsilon \log \epsilon}{N_{H}(1-\epsilon)+N_{L} \epsilon}
$$

When $\epsilon \rightarrow 0\left(o_{H} \rightarrow 1\right.$ and $\left.o_{L} \rightarrow 0\right)$, the entropy $H$ tends to $\log \left(N_{H}\right)$, as easily seen in the formula. In Figure 6 the entropy is plotted as a function of the number of active nodes $N_{H}$, for the cases $\epsilon=0,0.03,0.1$, and for $N=N_{H}+N_{L}=50$ as in our networks. The figure shows how the entropy of the output of a network trained between 0 and 1, given our assumptions, spans the whole range from 0 to $\log N$, while the network trained between $\epsilon$ and $1-\epsilon$ has a more limited range in the high entropy region. The range strongly depends on $\epsilon$. In our experiments one network was trained with $[0,1]$ targets and the other two with $[0.1,0.9]$ targets $(\epsilon=0.1)$.

In Section 5.4 the impact of $\epsilon$ on the entropy based confidence measure is discussed with examples on the test data.

\section{Data}

The experiments were performed on the Swedish SpeechDat corpus (Elenius, 2000), containing recordings of 5000 speakers over the telephone. The official training and test sets defined in SpeechDat and containing respectively 4500 and 500 speakers, were used in the experiments. Mel frequency cepstrum features were extracted at $10 \mathrm{~ms}$ spaced frames.

The training material for the neural networks, and the test material were restricted to the phonetically rich sentences. The training material was further 


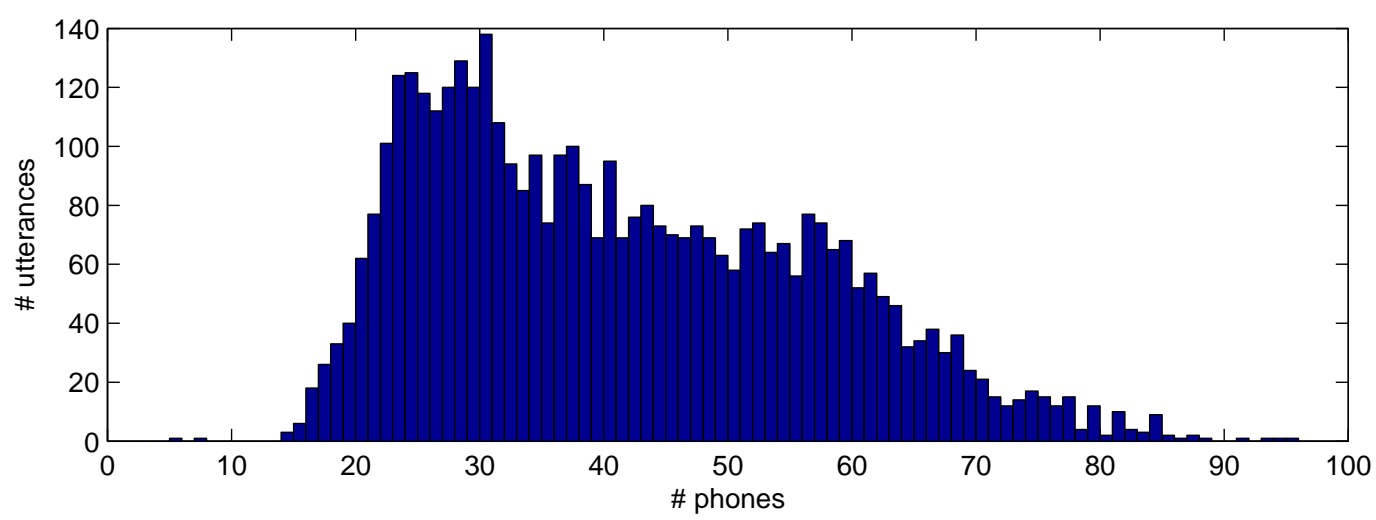

Figure 7. Distribution of the length of the test utterances in terms of number of phonemes.

divided into training and validation sets of 33062 and 500 utterances respectively. The test set contains 4150 utterances with an average length of 40.9 phonemes per utterance. Figure 7 shows the distribution of the length of the test utterances in terms of number of phonemes.

One problem with the SpeechDat database, that is important when training the MLPs and for testing at the frame level, is the unbalanced amount of silence frames compared to the amount of material for any phonetic class. Part of the silence frames that were concentrated at the beginning and at the end of each utterance, was removed.

Since the dataset lacks phonetic transcriptions, some pre-processing was necessary. The time-aligned reference, used for both training and testing the MLP models, was obtained with forced alignment employing the word level transcriptions, the official SpeechDat lexicon and triphonic HMMs based on Gaussian models. The HMMs were trained with the procedure defined in the RefRec scripts (Lindberg et al., 2000). The alignment lattice allowed non speech sounds to be inserted between words. The method proved to be reasonably accurate for our purposes.

\subsection{Acoustic Models}

Three multi-layer perceptrons were used in this study. The first (ANN) is a feed-forward network with two hidden layers of 400 fully connected units each. RNN1 and RNN2 are recurrent networks with one hidden layer of 400 units and a varying number of time delayed connections. The choice of topology in ANN aimed at ensuring a comparable complexity (in number of free parameters) between ANN and RNN1.

As a reference, results obtained with a set of Gaussian mixture models (GMM), 
Table 1

Details on the Acoustic Models and Frame-by-frame MAP results

\begin{tabular}{lccccc}
\hline \hline model & \# params & \# hidd.units & \# hidd.layers & recurrent & $\%$ correct frame \\
\hline GMM & 379050 & - & - & - & $35.4 \%$ \\
ANN & 186050 & 800 & 2 & no & $31.5 \%$ \\
RNN1 & 185650 & 400 & 1 & yes & $49.6 \%$ \\
RNN2 & 541250 & 400 & 1 & yes & $54.2 \%$ \\
\hline \hline
\end{tabular}

are reported together with the multi-layer perceptrons results. The GMM results show the discriminative power of the set of Gaussian models, when the phonetic class with the maximum a posteriori probability (MAP) is selected for each frame. The Gaussian mixture parameters where estimated using the HMM training with 32 Gaussian terms per state.

Details on the acoustic models are reported in Table 1 . The table shows the overall number of parameters, and, in the case of the perceptrons, the number of hidden layers and hidden units and the dynamic characteristics. The last column reports the correct frame classification rate when the maximum $a$ posteriori class was selected frame-by-frame.

\subsection{Implementation note}

The HMM training was performed using the HTK Toolkit (Young et al., 2002). The MLP training algorithm was implemented in the NICO Toolkit (Ström, 1996). The modified Viterbi algorithm is the decoder used in the SYNFACE project (Salvi, 2003; Karlsson et al., 2003), and, together with the other tools used in the experiments, was implemented by the author. The statistical analysis was performed using the $\mathrm{R}$ software ( $\mathrm{R}$ Development Core Team, 2003). All experiments were performed on a GNU-Linux platform running on standard hardware (PC).

\section{Results}

Results obtained with a normal phone loop are reported in Figure 8 as a reference to the performance of the recogniser in the real task. The left plot in the figure shows the average correct frame classification rate over the 4150 test utterances for varying look-ahead length and for the three neural networks (vit-ANN, vit-RNN1 and vit-RNN2). The horizontal lines in the figure indicate the classification rate without Viterbi decoding, i.e. selecting the highest 

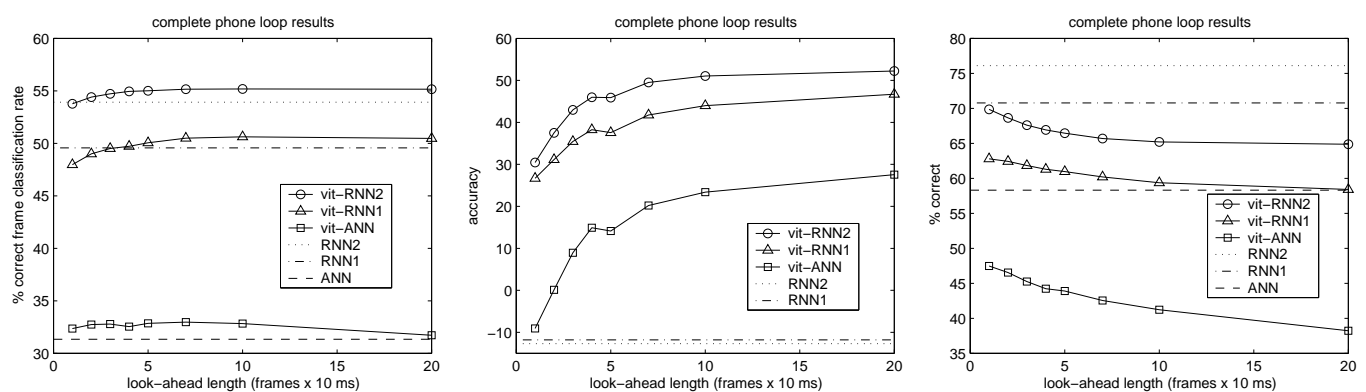

Figure 8. Phone loop results
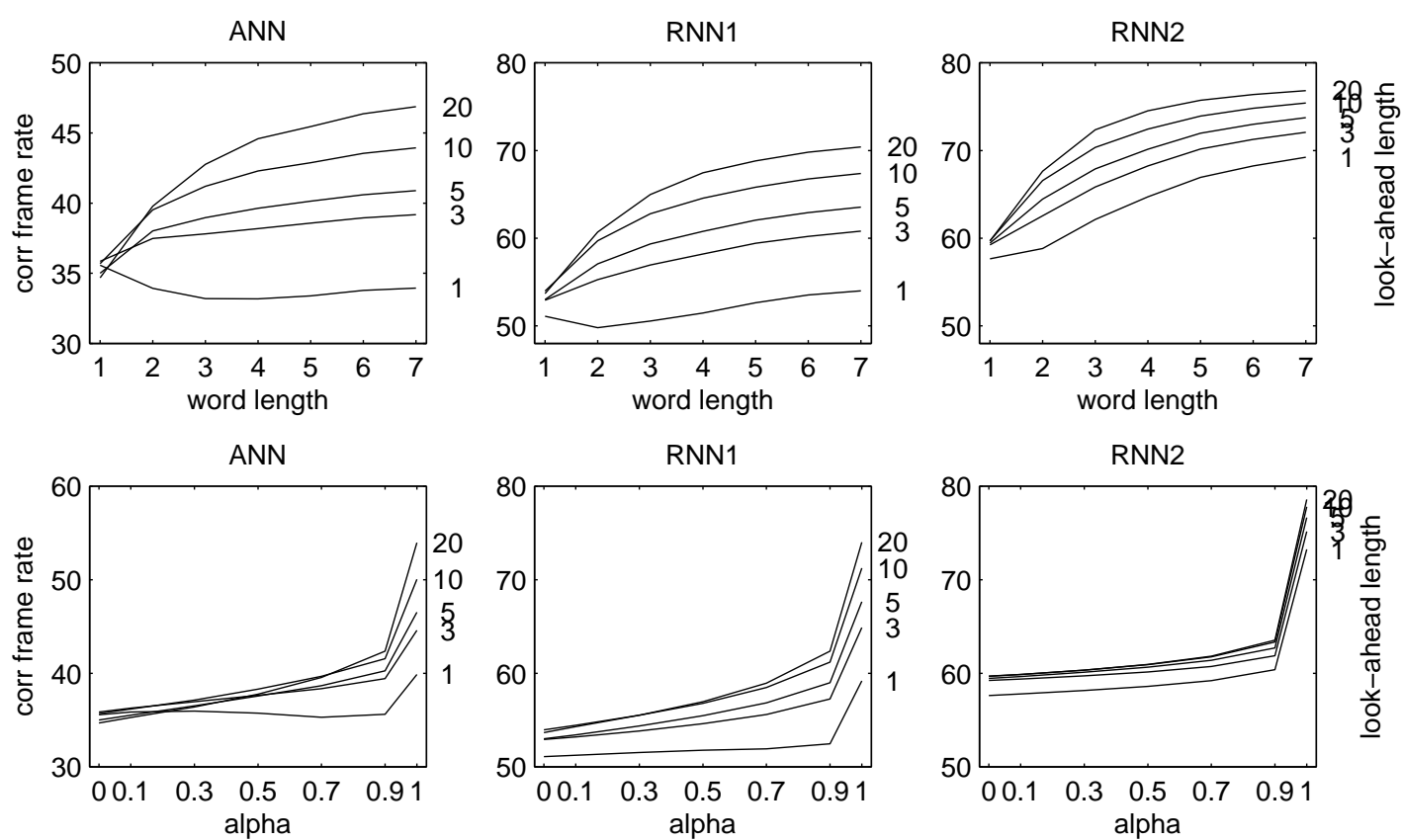

Figure 9. Summary of "wordlen" (top) and "alpha" (bottom) results

activity output at each frame (frame-by-frame maximum a posteriori). The results are very close to the ones obtained in Salvi (2003), the differences being due to the way the boundary effects are handled (see Section 3.3), and to the fact that in Salvi (2003) a global score was computed over the whole test material, while here we compute the correct frame classification rate of each utterance and then average the results.

The middle and right plots in Figure 8 show the accuracy and percent of correct words as defined in (Young et al., 2002). These results are reported in order to compare the three scoring methods, and to mention that none of them are fully satisfying given the application. Accuracy and correct words do not take into account segment boundary alignment in time and were therefore discarded in the following evaluation. Correct frame classification rate, in contrast, does not indicate how stable the result is (number of symbol insertions).

The "wordlen" and "alpha" tests results are summarised in Figure 9. In the 
first case (top) the average correct frame rate is plotted as a function of the word length in the "wordlen" test for different values of the look-ahead length and for the three multi-layer perceptrons. In the second case (bottom) the $x$-axis indicates the $\alpha$ parameter in the "alpha" test. Note that the range of the $y$-axis in the ANN case is different from the other two.

Longer time dependencies in the language model (LM) and longer look-ahead lengths are beneficial in most conditions, as most of the curves increase monotonically and do not overlap. Exceptions to this are the conditions in which the static model ANN is used in conjunction with either a short time LM or a short look-ahead length. In those cases, more irregular results can be found (see left plots in the Figure). Examples are the fluctuating results corresponding to different look-ahead lengths when wordlen $=1$ or $\alpha=0$ (top-left and bottom-left plots) and the non-monotonic variation of the score with respect to the word length and $\alpha$ when the look-ahead length is one frame (top-left and bottom-left plots). The last phenomenon can be found also in the RNN1 case (top-middle plot).

In the following, these results will be analysed in details with statistical means.

\subsection{Wordlen test: short to long time dependencies}

Figure 10 (top) shows box plots for the phone-loop case (word length $=1$ ) for different look-ahead lengths. The middle line in the box displays the median, while the lower and higher lines, called lower and higher hinges, display respectively the first and third quartiles. The lower and higher whiskers show the full range of the data. It is not clear in the ANN case, whether the use of longer look-ahead is beneficial to the recognition score. In RNN1 and RNN2 there is a slight improvement along increasing look-ahead lengths. Applying an ANOVA to the data returns significant differences in all the three cases (respectively for ANN, RNN1 and RNN2: $\mathrm{F}(4,20745)=15.31,21.79,8.96 ; \mathrm{p}=$ $\left.1.65 \times 10^{-12},<2.2 \times 10^{-16},<3.1 \times 10^{-7}\right)$. A successive Tukey multiple comparison test is visualised in Figure 10 (bottom). The figure indicates the 95\% familywise intervals for all possible combination of the values of the look-ahead factor. The difference between condition $x$ and $y$ is indicated by $x-y$ that is the increase in correct frame rate going from look-ahead length $y$ to $x$. If an interval crosses the zero line, the differences between the two conditions are not significant.

There are significant differences in ANN but with negative signs. In RNN1 the difference $10-3$ and all differences between $L=1$ and $L \neq 1$ are significant. Finally in RNN2 only the $L=1$ condition seems to be distinguishable from the others. 

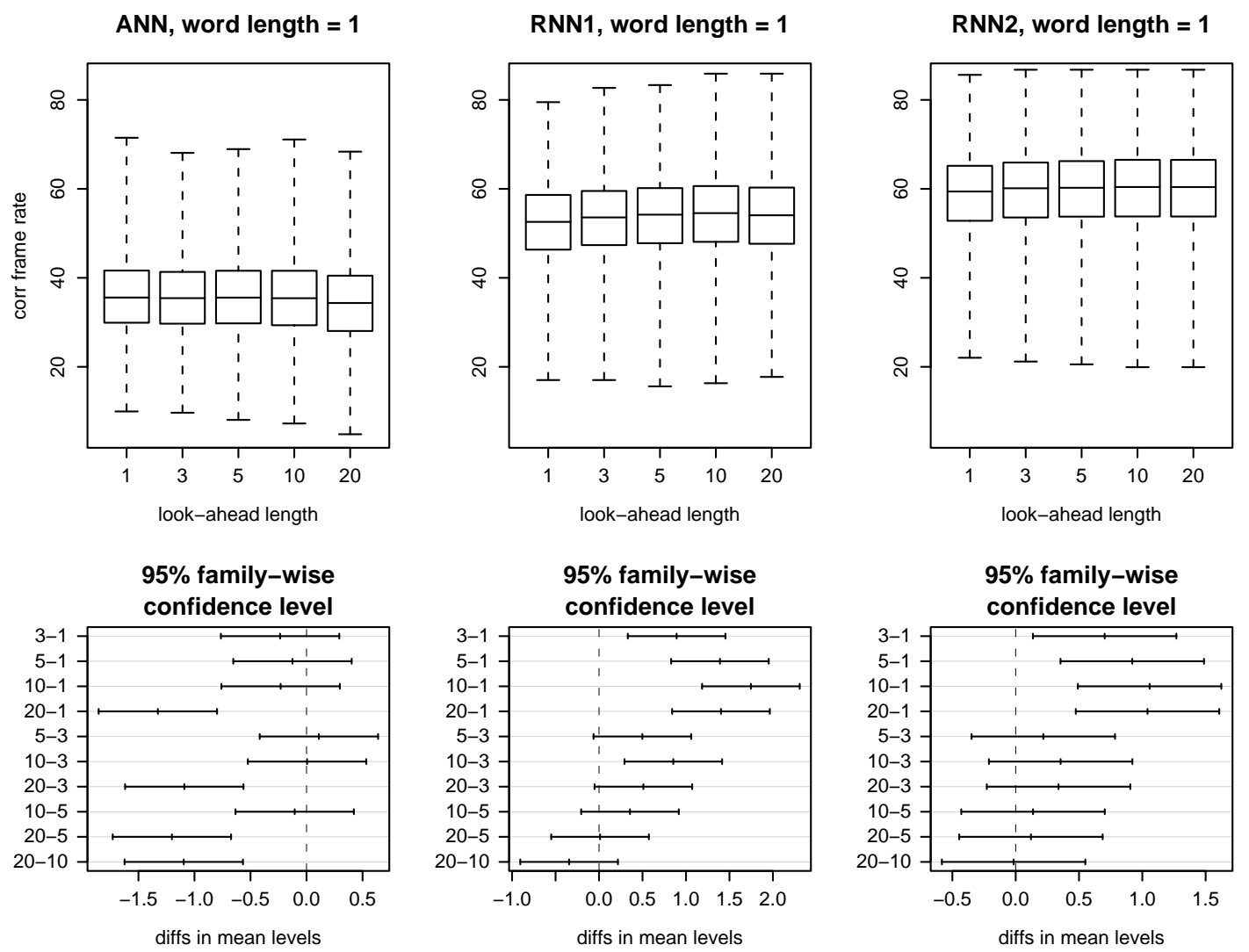

Figure 10. Box-plots (top) and 95\% family-wise Tukey confidence intervals (bottom), word length $=1$

On the other end of the word length parameter values (wordlen $=7$ ), the information carried by the transition model, and the Viterbi processing has a stronger effect on the feed-forward perceptron. Figure 11 (top) shows the corresponding box plot. The differences are significant in all cases (respectively for ANN, RNN1 and RNN2: $\left.\mathrm{F}(4,20745)=281.32,707.16,262.17 ; \mathrm{p}=<2.2 \mathrm{x} 10^{-16}\right)$. Multiple comparison leads to significance in every difference as illustrated by Figure 11 (bottom). Of the consecutive distances, $3-1$ is always the greatest. In ANN and RNN1, $10-5$ is greater than $5-3$.

Table 2 summarises the results for intermediate word lengths. A plus sign in a $x-y$ column indicates a positive significant difference between the latency conditions y and $\mathrm{x}$, a minus sign indicates a significant negative difference and, finally, "n" no significant difference.

\subsection{Alpha test: short to long time dependencies}

Experiments carried out with the "alpha" test show similar results to the "wordlen" test. In the phone loop condition $(\alpha=0.0)$ the language model is 

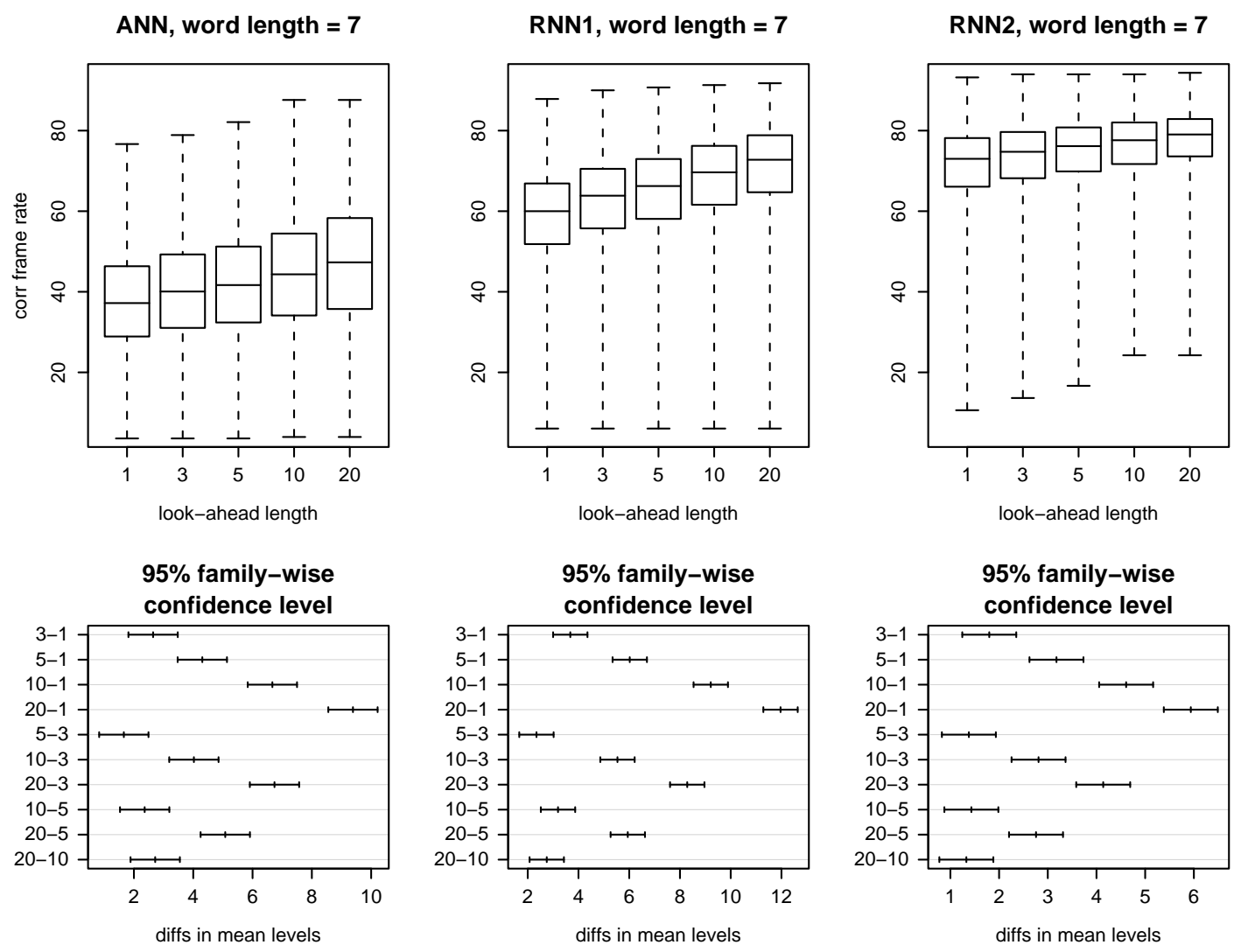

Figure 11. Box-plots (top) and 95\% family-wise Tukey confidence intervals (bottom), word length $=7$

Table 2

Wordlen test: Tukey HSD multiple comparison results ANN

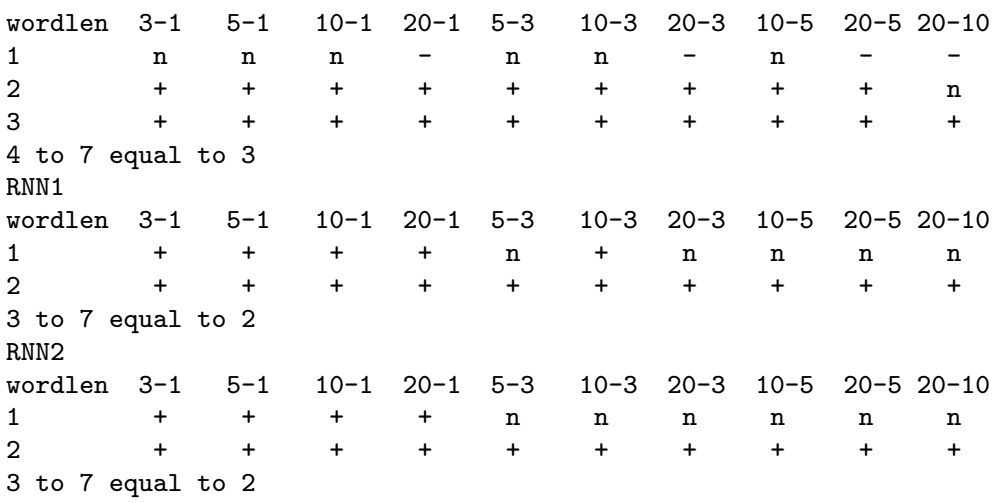

equivalent to the one in the "wordlen" test with word length 1 (see previous section). Figure 12 shows the intermediate condition $\alpha=0.5$. In the ANN case the $3-1,5-1$ and $10-1$ differences are significant and positive, while the $20-$ 10 difference is significant but negative. RNN2 shows clear significant differences when changing from 1 frame to longer look-ahead. The $5-3$ and $10-3$ differences are also significant but less evidently. With RNN1 all differences are significant except $20-10$. 

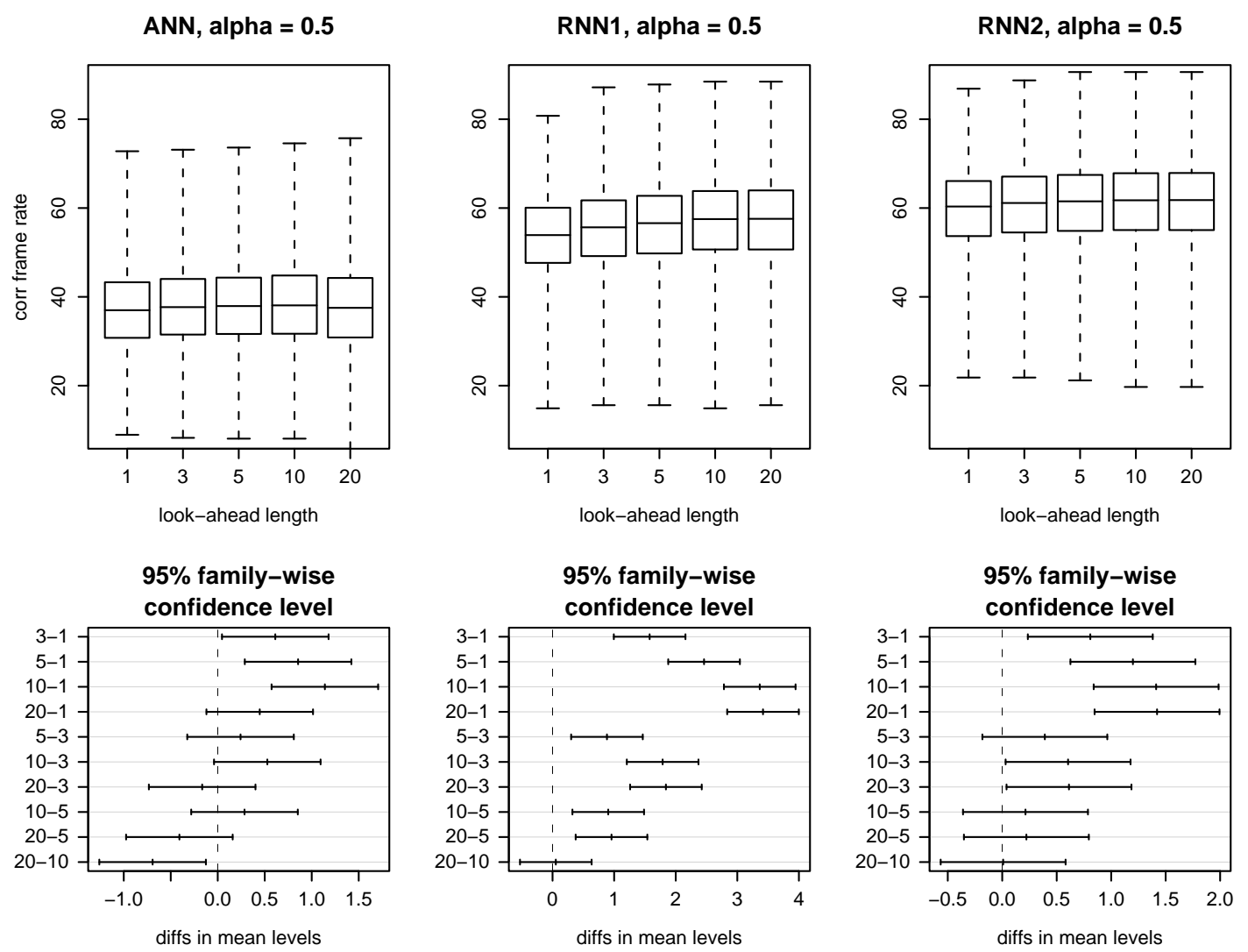

Figure 12. Box-plots (top) and 95\% family-wise Tukey confidence intervals (bottom), alpha $=0.5$

For $\alpha=1$, the LM specifies forced alignment. The results in Figure 13 indicate significant increase of the correct frame classification rate with respect to the look-ahead length, in all cases. Finally, Table 3 shows the Tukey results in all intermediate cases. These are not as regular as the "wordlen" results, revealing differences between the neural networks.

\subsection{Summary}

In both the "wordlen" and "alpha" tests, the statistic analysis shows significant differences. A successive Tukey multiple comparison test shows which couples of values of the look-ahead parameter correspond to significant differences in the correct frame rate. The results strongly depend on the MLP for short time dependences in the recognition network (towards the phone loop condition). This dependency fades when the condition is shifted towards the forced alignment case. 

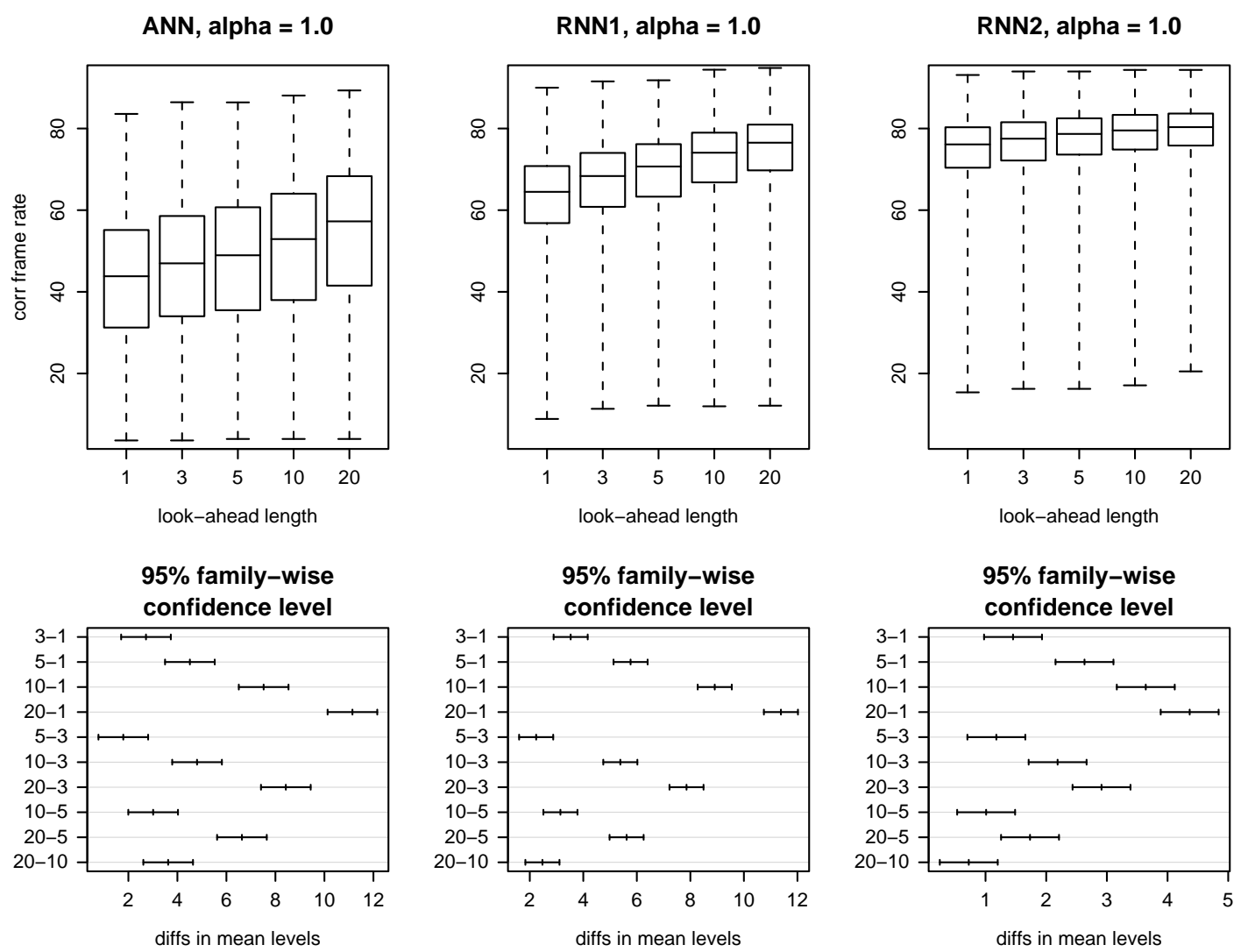

Figure 13. Box-plots (top) and 95\% family-wise Tukey confidence intervals (bottom), alpha $=1.0$

Table 3

Alpha test: Tukey HSD multiple comparison results

$\begin{array}{lcccccccccc}\text { alpha } & 3-1 & 5-1 & 10-1 & 20-1 & 5-3 & 10-3 & 20-3 & 10-5 & 20-5 & 20-10 \\ 0.0 & \mathrm{n} & - & \mathrm{n} & - & - & \mathrm{n} & - & + & \mathrm{n} & - \\ 0.1 & \mathrm{n} & \mathrm{n} & \mathrm{n} & - & \mathrm{n} & \mathrm{n} & - & \mathrm{n} & - & - \\ 0.3 & \mathrm{n} & \mathrm{n} & \mathrm{n} & \mathrm{n} & \mathrm{n} & \mathrm{n} & - & \mathrm{n} & - & - \\ 0.5 & + & + & + & \mathrm{n} & \mathrm{n} & \mathrm{n} & \mathrm{n} & \mathrm{n} & \mathrm{n} & - \\ 0.7 & + & + & + & + & \mathrm{n} & + & + & + & \mathrm{n} & \mathrm{n} \\ 0.9 & + & + & + & + & \mathrm{n} & + & + & + & + & \mathrm{n} \\ 1.0 & + & + & + & + & + & + & + & + & + & + \\ \text { RNN1 } & & & & & & & & & & \\ \text { alpha } & 3-1 & 5-1 & 10-1 & 20-1 & 5-3 & 10-3 & 20-3 & 10-5 & 20-5 & 20-10 \\ 0.0 & + & + & + & + & \mathrm{n} & + & + & + & + & \mathrm{n} \\ 0.1 & + & + & + & + & \mathrm{n} & + & + & \mathrm{n} & \mathrm{n} & \mathrm{n} \\ 0.3 & + & + & + & + & + & + & + & + & + & \mathrm{n} \\ 0.5 & + & + & + & + & + & + & + & + & + & \mathrm{n} \\ 0.7 & + & + & + & + & + & + & + & + & + & \mathrm{n} \\ 0.9 & + & + & + & + & + & + & + & + & + & + \\ 1.0 & + & + & + & + & + & + & + & + & + & + \\ \text { RNN2 } & & & & & & & & & & \\ \text { alpha } & 3-1 & 5-1 & 10-1 & 20-1 & 5-3 & 10-3 & 20-3 & 10-5 & 20-5 & 20-10 \\ 0.0 & + & + & + & + & \mathrm{n} & \mathrm{n} & \mathrm{n} & \mathrm{n} & \mathrm{n} & \mathrm{n} \\ 0.1 & + & + & + & + & \mathrm{n} & \mathrm{n} & \mathrm{n} & \mathrm{n} & \mathrm{n} & \mathrm{n} \\ 0.3 & + & + & + & + & \mathrm{n} & \mathrm{n} & \mathrm{n} & \mathrm{n} & \mathrm{n} & \mathrm{n} \\ 0.5 & + & + & + & + & \mathrm{n} & + & + & \mathrm{n} & \mathrm{n} & \mathrm{n} \\ 0.7 & + & + & + & + & \mathrm{n} & + & + & \mathrm{n} & \mathrm{n} & \mathrm{n} \\ 0.9 & + & + & + & + & + & + & + & \mathrm{n} & + & \mathrm{n} \\ 1.0 & + & + & + & + & + & + & + & + & + & + \\ & & & & & & & & & & +10\end{array}$



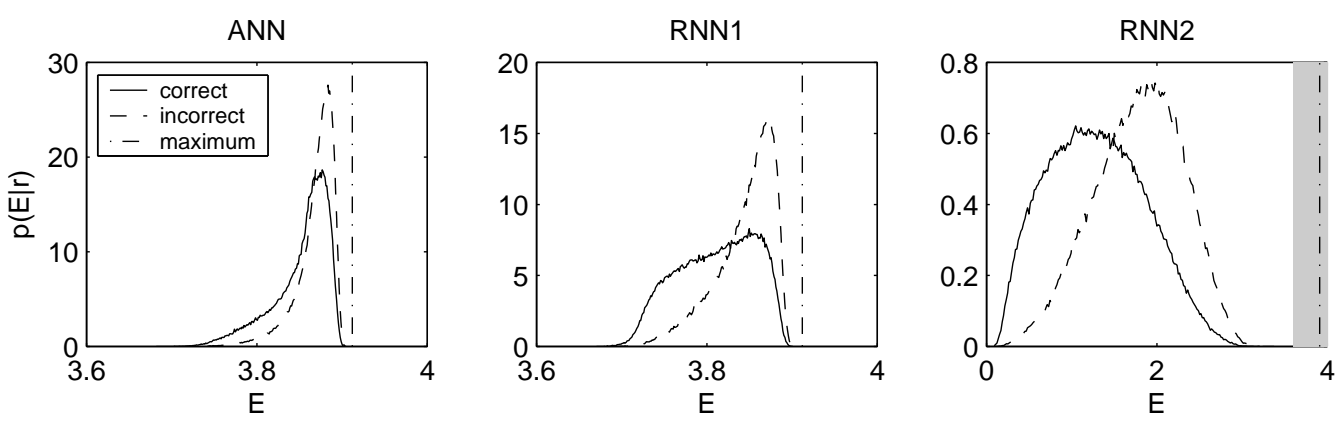

Figure 14. Entropy distribution for correct and incorrect classification

Table 4

Prediction capabilities of the entropy as confidence measure (ML decision)

\begin{tabular}{l|cc|cc|c}
\hline \hline net & corr. accept & corr. reject & false accept & false reject & tot. error \\
\hline ANN & $21.5 \%$ & $37.2 \%$ & $10.2 \%$ & $31.1 \%$ & $41.3 \%$ \\
RNN1 & $32.1 \%$ & $34.2 \%$ & $17.7 \%$ & $16.0 \%$ & $33.8 \%$ \\
RNN2 & $32.4 \%$ & $33.9 \%$ & $21.8 \%$ & $11.9 \%$ & $33.7 \%$ \\
\hline \hline
\end{tabular}

\subsection{Confidence measure}

Figure 14 shows the distribution of the entropy for correct (continuous line) and incorrect (dashed line) classification, and for the networks ANN (left), RNN1 (centre), and RNN2 (right). The vertical dashed-dotted lines indicate the maximum entropy $(\log N)$. In the rightmost plot, the shaded area corresponds to the range chosen in the other two plots, and is used to facilitate the comparison between the two conditions. For the networks trained with $[0.1,0.9]$ targets (ANN and RNN1) the entropy is concentrated in the high range, as explained in Section 3.5. For RNN2 the entropy range is larger (the network was trained with $[0,1]$ targets).

The prediction capabilities of the entropy as confidence measure are however very similar for the recurrent networks. If we consider a Maximum Likelihood decision, based on the conditional entropy distributions, that leads to the minimum total error in case of equal a priori probabilities, we obtain the results shown in Table 4. Note that the distributions shown in Figure 14 and the results in Table 4 are obtained on two independent data sets created by splitting the test data into two subsets of equal size. 


\section{Discussion}

The three factors considered in the experiments seem to strongly interact in the decoding process. When the language model (LM) is similar to a phone loop, most of the information on the time evolution is provided by the multi-layer perceptron. In this case differences emerge on the latency behaviour of different neural network topologies. The static network (ANN) produces irregular results when the look-ahead length $L$ is varied. The dynamic models (RNN1 and RNN2) show a slight improvement with increasing $L$, that fades for higher values of $L$. The look-ahead length for which no further improvement is achieved seems to be lower for RNN2 than for RNN1.

When the LM contains longer time dependencies, all acoustic models benefit (to different extents) of longer look-ahead lengths. This can be explained by noting that

- the Viterbi decoding makes use of time dependent information regardless of its source (transition model or dynamic neural network),

- the information provided by the transition model and the dynamic neural network might overlap/conflict,

- the look-ahead length needed to take full advantage of the Viterbi decoding is closely related to the length of the time correlations contained in the hybrid model (transition model or dynamic neural network).

Given these remarks, the results obtained here can be interpreted in the following way. The static model ANN, providing no time dependent information, takes advantage of the Viterbi decoding only for long time transition models and long look-ahead. The more complex recurrent perceptron (RNN2) provides information that partly overlaps with the transition model, causing only limited improvements when the look-ahead is increased (especially in the "alpha" test). The simpler recurrent perceptron (RNN1) provides more limited time dependent information and takes more advantage of the Viterbi decoding.

However, more specific tests should be designed to support this interpretation, using, for example, techniques from non-linear dynamics to analyse the dynamical behaviour of the recurrent networks in details. Factors such as the target values during training should also be excluded from the tests. The scoring method used rises also questions on the kind of errors the system is affected by in different conditions. It would be important, for example, to investigate to which extent the errors are due to misclassification of isolated frames or longer sequences, or to misalignment of the segment boundaries. 


\section{Conclusions}

The interaction of transition model, dynamic probability estimators and lookahead length in the decoding phase of a speech recognition system has been analysed in this paper. The results show how the dynamic information provided by the recurrent multi-layer perceptrons does not always interact in a constructive way with the transition model in Viterbi decoding. With static MLPs, the use of longer look-ahead lengths is not beneficial when the time dependencies in the language model are limited as in the phone loop condition. With recurrent MLPs, the benefit depends on the complexity of the network.

The frame-by-frame entropy proved to be a reasonably accurate confidence measure. This measure is not strongly affected by the use of target values in training other than $[0,1]$.

\section{Acknowledgements}

This research was funded by the Synface European project IST-2001-33327 and carried out at the Centre for Speech Technology supported by Vinnova (The Swedish Agency for Innovation Systems), KTH and participating Swedish companies and organisations.

\section{References}

Beskow, J., 2004. Trainable articulatory control models for visual speech synthesis. Journal of Speech Technology 7 (4), 335-349.

Bourlard, H., Morgan, N., Nov. 1993. Continuous speech recognition by connectionist statistical methods. IEEE Trans. Neural Networks 4 (6), 893-909.

Elenius, K., 2000. Experience from collecting two Swedish telephone speech databases. International Journal of Speech Technology 3, 119-127.

Imai, T., Kobayashi, A., Sato, S., Tanaka, H., Ando, A., 2000. Progressive 2-pass decoder for real-time broadcast news captioning. In: ICASSP. pp. 1937-1940.

Karlsson, I., Faulkner, A., Salvi, G., 2003. SYNFACE - a talking face telephone. In: Proc. Eurospeech. pp. 1297-1300.

Kwan, D., Kallel, S., May 1998. A truncated best-path algorithm. IEEE Trans. Commun. 46 (5), 565-567.

Lindberg, B., Johansen, F. T., Warakagoda, N., Lehtinen, G., Kačič, Z., Z̆gank, A., Elenius, K., Salvi, G., 2000. A noise robust multilingual reference recogniser based on SpeechDat(II). In: 6th Intern. Conf. on Spoken Language Processing. Vol. III. pp. 370-373. 
Ljolje, A., Hindle, D. M., Riley, M. D., Sproat, R. W., May 2000. The AT\&T LVCSR-2000 system. In: Speech Transcription Workshop. University of Maryland.

R Development Core Team, 2003. R: A language and environment for statistical computing. R Foundation for Statistical Computing, Vienna, Austria, ISBN 3-900051-00-3.

URL http://www.R-project.org

Robinson, A. J., Mar. 1994. An application of recurrent nets to phone probability estimation. IEEE Trans. Neural Networks 5 (2), 298-304.

Robinson, A. J., Cook, G. D., Ellis, D. P. W., Fosler-Lussier, E., Renals, S. J., Williams, D. A. G., 2002. Connectionist speech recognition of broadcast news. Speech Communication 37, 27-45.

Salvi, G., 2003. Truncation error and dynamics in very low latency phonetic recognition. In: ISCA Tutorial and Research Workshop on Non-linear Speech Processing (NOLISP), Le Croisic, France.

Ström, N., Oct.-Dec. 1992. Development of a recurrent time-delay neural net speech recognition system. TMH-QPSR 26 (4), 1-15.

Ström, N., 1996. The NICO Toolkit for Artificial Neural Networks. Dept. for Speech, Music and Hearing, Stockholm, Sweden.

URL http://www.speech.kth.se/NICO

Viterbi, A. J., Apr. 1967. Error bounds for convolutional codes and an asymtotically optimum decoding algorithm. IEEE Trans. Inform. Theory IT-13, 260-269.

Weathers, A. D., 1999. An analysis of the truncated Viterby algorithm for PRML channels. In: Proc. IEEE Intern. Conf. on Comm. Vol. 3. pp. 19511956.

Werbos, P. J., Oct. 1990. Backpropagation through time: what it does and how to do it. Proc. of the IEEE 78 (10), 1550-1560.

Williams, G., Renals, S., 1999. Confidence measures from local posterior probability estimates. Computer Speech and Language 13 (4), 395-411.

Young, S., Evermann, G., Kershaw, D., Moore, G., Odell, J. J., Ollason, D., Valtchev, V., P. C. Woodland, P., 2002. The HTK Book, Version 3.2. Cambridge University Engineering Department. 